\title{
Detection of Extended spectrum beta lactamases in gram negative isolates causing urinary tract infection in Tertiary care centre
}

\author{
M. D. Khaleel ${ }^{1}$, Rozina Arshi Khan ${ }^{2}$, Syeda Daniya Samreen ${ }^{3, *}$, Syeda Juvaria Arshi ${ }^{4}$ \\ ${ }^{1}$ Professor, ${ }^{2}$ Senior Resident, ${ }^{3}$ Final year Medical Student, ${ }^{4}$ Intern, ${ }^{1,2}$ Dept. of Microbiology, Deccan College of Medical \\ Sciences, Kanchan Bagh, Warangal, Telangana, India
}

*Corresponding Author:

Email: dan.samreen@gmail.com

\begin{abstract}
Introduction: Urinary Tract infection (UTI's) is the most commonly encountered infectious disease, affecting both the sexes in all age groups. This leads to consumption of large number of antibiotics and development of resistant strains leading to complications.

Objective: The study was carried out to know the distribution pattern and to evaluate the sensitivity of ESBL producing urinary isolates from patients with UTI.

Materials and Methods: A cross sectional study was conducted at Owaisi Hospital and Research Centre for 6months. A total of 392 samples were obtained from suspected UTI patients. Clean catch mid stream urine samples obtained were subjected to microscopy, culture and antibiotic sensitivity testing as per CLSI guidelines.

Results: From the total of 392 samples, 200 samples i.e. 51\% samples were culture positive. Among the culture positive cases, $69 \%$ were females, $35 \%$ were males. $38.8 \%$ of Esch.coli and $25 \%$ Klebsiella were predominant ESBL producers.

Conclusion: This study revealed that majority of Gram negative urinary isolates are ESBL producers, and multidrug resistant. Thus strict policy with guidelines for antibiotic susceptibility testing and prescription should be followed to prevent further emergence of resistant strains and treatment failure.
\end{abstract}

Keywords: Mid stream urine, Extended Spectrum Beta Lactamases, antibiotic susceptibility pattern.

\section{Introduction}

Urinary tract infection (UTI) is one of the most commonly encountered infectious disease. ${ }^{1}$ UTIs affect almost all age groups and diagnosed in both hospitalized and outpatients.This infection not only causes a serious burden on the socioeconomic life of individuals but also leads to the consumption of a large proportion of all antibacterial drugs used in the world. ${ }^{2}$ Women are more susceptible to UTIs than men due to anatomical structure of their genitourinary tract. ${ }^{3}$

UTI is most commonly caused by bacteria from patients own intestinal flora that enters urinary tract by ascending route via the urethra. It is an illness that can occur from infancy through old age, in otherwise healthy persons and in those who are compromised or debilitated. In fact, UTI are most common group of acute infection observed in nursing home residence and are most frequent cause of bacteraemia in patients with indwelling catheters and in institutional setting. They account for $>40 \%$ of all institutionally acquired infections. $^{2}$

Gram negative bacilli account for majority of UTI, specifically Esch.coli (upto85\%), Klebsiella, Proteus and Pseudomonas aeruginosa. ${ }^{1}$ Among gram positive cocci Staphylococcus saprophyticus and Enterococcus species are major etiological agents. ${ }^{6}$

The Gram negative urinary isolates producing ESBLs are those that hydrolyze the oxyimino betalactams and monobactams, but have no effect on the cephamycins and carbapenems.Problems associated with
ESBL producing isolates are difficult to be detected or treated, thereby causing increased mortality.

Antibiotic susceptibility testing should be performed on isolates from symptomatic patients with bacteriuria and colony counts that are clinically significant. Each laboratory should decide which antibiotics to test and report after consultation with infectious disease practitioners and pharmacy staff. Routine susceptibility testing for most antibiotics should be standardized and interpretation of results should be based on anticipated responses to infection. Thus delay in appropriate treatment leads to complications. ${ }^{7}$ Therefore, timely usage of antimicrobial agents discourages the indiscriminate usage of antibiotics and also decreases the hospital stay.

\section{Objective}

The current study is being under taken with the following objectives:

1. To isolate and detect gram negative urinary isolates.

2. To determine antibiotic sensitivity among isolated gram negative bacteria.

3. To detect ESBL producing bacterial isolates among resistant gram negative bacteria.

\section{Materials and Methods}

A cross sectional study, was carried out among patients who attended OP and IP at Owaisi Hospital during August 2016 - January 2017. Patients were referred from departments of Urology, Nephrology and Intensive care units. Patients who presented with 
symptoms consistent with cystitis or urethritis underwent a thorough history, physical examination and urine analysis. However, urine culture continues to be important in patients with recurrent UTI or treatment failure.

In view of increasing antimicrobial resistance in urinary pathogens, culture is necessary for performing antimicrobial susceptibility testing. Appropriate collection of microbiology urine specimens, has an important influence on usefulness of culture result. Collection of clean catch mid stream urine specimen, is the most frequently used and preferred method of urine collection because it is non invasive and avoids the risks inherent to catheterization.

\section{Selection criteria}

Inclusion criteria:

1. Patient with symptom of urinary tract infection \& not started on any antibiotic therapy.

2. Both male and females of age $10-60$ yrs will be included.

\section{Exclusion criteria:}

1. Asymptomatic patients

2. Prior antibiotic therapy

3. Pregnant women.

Specimen collection and processing: A single clean voided specimen or a single specimen obtained by catheterization in a patient with specific symptoms was taken, if significant pyuria is demonstrable and culture yields a recognisable uropathogen. For purposes of optimal quantitation, it has been recommended by guidelines that only first morning specimens be processed or, if such specimens are not available, that the urine be allowed to incubate in the bladder for as long as possible (with a minimum of 4 hours) before collection to increase the bacterial density. Quantitation is necessary for the diagnosis of asymptomatic bacteriuria but unnecessary in case of symptomatic patients. Urine specimens from other asymptomatic patient populations, except those demonstrating significant pyuria, could not be reliably interpreted.

Specimen Transport: Urine is an excellent supportive medium for the growth of most uropathogens and therefore must be immediately received, refrigerated and processed in the laboratory within 2 hours. Longer delays render examination for significant pyuria unreliable, and the extremes of $\mathrm{pH}$ and urea concentration and the presence of antimicrobial agents may adversely affect the recovery of uropathogens.

Culture: All of the samples were Mid stream urine specimens and then they were subjected to culture. Generally, routine culture includes plating onto one nonselective medium. Calibrated loops of $0.01 \mathrm{ml}$ were used, not $0.001 \mathrm{ml}$ loops, as quantitation is difficult to obtain with low inoculums. Specimens were routinely inoculated on a blood agar plate, a MacConkey agar plate and CLED medium and were incubated for 48 hours.
Specimens with multiple uropathogens (i.e., three or more) indicate probable contamination. Susceptibility test were performed for inpatients. Outpatient cases were subjected to different algorithm that does not routinely calls for susceptibility test, rather it emphasizes empirical selection based on antibiogram. Culture was considered positive if the specimen showed a single organism at concentration of $\geq 10^{5} \mathrm{CFU} / \mathrm{ml}$ or one or two uropathogens present in small number (i.e. $\geq 10^{2} \mathrm{CFU} / \mathrm{ml}$ ) or, $\left(. \geq 10^{2}\right.$ to $\left.<10^{5} \mathrm{CFU} / \mathrm{ml}\right)$ in clinical situation such as in acute urethral syndrome or cases of previous antibiotic theory $^{8}$.

Microorganisms were identified as gram negative or positive by gram strain and standard biochemical tests.

\section{Susceptibility Reporting}

With the growing number of emerging uropathogens and the simultaneous increase in newer antibiotics, it is mandatory that we use standardized methods and report only appropriate antibiotics for UTIs. Antibiotic susceptibility testing was done by Kirby Bauer disc diffusion test. Antimicrobial agents approved by the Food and Drug administration ( FDA) for routine testing and reporting by clinical microbiology laboratories for urinary tract isolates are listed as Group U supplementary for urine only in the 2006 CLSI Performance standards for Antimicrobial Disc susceptibility test were used. ${ }^{10}$

\section{ESBL Detection:}

\section{Phenotypic method}

a. Screening test

b. Confirmatory method

Screening Test (Kirby Bauer Disc Diffusion Test) : Materials:

1. Mueller Hinton agar.

2. Test strain standardized inoculums (0.5 McFarland turbidity)

3. Control strains

4. Antibiotic discs of $30 \mu \mathrm{g}$ Ceftazidime $30 \mu \mathrm{g}$ Cefotaxime $30 \mu \mathrm{g}$ Ceftriaxone

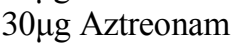

\section{Method:}

1. Lawn culture the standardized inoculums of test isolate ( 0.5 McFarland turbidity) on Mueller Hinton agar plate.

2. Place $30 \mu \mathrm{g}$ disc each of ceftazidime, cefotaxime, ceftriaxone and aztreonam and $10 \mu \mathrm{g}$ of cefpodoxime.

3. Incubate for $16-18$ hours at $37^{\circ} \mathrm{C}$.

Results: As per the guidelines issued by CLSI, Klebsiella.pneumoniae, K. oxytoca, E. coli, and P. mirabi lis isolates were regarded as positive for screening test under the following conditions. 
Table 1: Screening by Disc diffusion method

\begin{tabular}{|c|c|c|}
\hline \multirow[t]{2}{*}{ Antibiotic } & \multicolumn{2}{|c|}{ Zone diameters when testing } \\
\hline & $\begin{array}{c}\text { K.pneumoniae, } \\
\text { K.oxytoca, } \\
\text { Esch.coli }\end{array}$ & P.mirabilis \\
\hline Cefpodoxime $10 \mu \mathrm{g}$ & $\leq 17 \mathrm{~mm}$ & $\leq 22 \mathrm{~mm}$ \\
\hline Ceftazidime $30 \mu \mathrm{g}$ & $\leq 22 \mathrm{~mm}$ & $\leq 22 \mathrm{~mm}$ \\
\hline Cefotaxime $30 \mu \mathrm{g}$ & $\leq 27 \mathrm{~mm}$ & $\leq 27 \mathrm{~mm}$ \\
\hline Ceftriaxone $30 \mu \mathrm{g}$ & $\leq 25 \mathrm{~mm}$ & \\
\hline Aztreonam $30 \mu \mathrm{g}$ & $<27 \mathrm{~mm}$ & \\
\hline
\end{tabular}

ESBL Confirmatory methods

i. Double Disc Synergy Test (DDST)/ Double Disc Diffusion Test (DDDT)

\section{Materials:}

1. Mueller Hinton agar

2. Test strain standardized inoculums $(0.5$ McFarland turbidity)

3. Control strains

4. Antibiotic discs

$30 \mu \mathrm{g}$ ceftazidime,

$30 \mu \mathrm{g}$ cefotaxime,

Amoxicillin/clavulanic acid 30/10 $\mu \mathrm{g}$

\section{Method:}

1. Lawn culture the standardized inoculums of test isolate (0.5 McFarland turbidity) on Mueller Hinton agar plate

2. An amoxicillin/clavulanic acid $30 \mu / 10 \mu \mathrm{g}$ is placed at the center of the plate.

3. Discs containing $30 \mu \mathrm{g}$ ceftazidime, cefotaxime, are placed 20-30 mm away from the central disc.

4. Incubate for $16-18$ hours at $37^{\circ} \mathrm{C}$

Result: An extension in the zone of inhibition around the peripheral discs towards the centrally placed Amoxicillin/clavulanic acid 30/10 $\mu \mathrm{g}$ disc indicates ESBL production.

ii CLSI Phenotypic Confirmatory Test (PCT)/ Combined Disc Method

Materials:

1. Mueller Hinton agar
2. Test strain standardized inoculums $(0.5$ McFarland turbidity)

3. Control strains

4. Antibiotics discs Ceftazidime $(30 \mu \mathrm{g})$, ceftazidime/clavulanic acid $(30 / 10 \mu \mathrm{g})$, cefotaxime $(30 \mu \mathrm{g})$, cefotaxime/clavulanic acid $(30 / 10 \mu \mathrm{g})$.

\section{Method:}

1. Lawn culture the standardized inoculums of test isolate ( 0.5 McFarland turbidity) on Mueller Hinton agar plate

2. Ceftazidime $(30 \mu \mathrm{g})$,ceftazidime/clavulanic acid $(30 / 10 \mu \mathrm{g})$, cefotaxime $(30 \mu \mathrm{g})$, cefotaxime/clavulanic acid $(30 / 10 \mu \mathrm{g})$ disks are placed on the plate $20 \mathrm{~mm}$ apart

3. Incubate for $16-18$ hours at $37^{\circ} \mathrm{C}$

Result: An increase in the zone diameter by $\geq 5 \mathrm{~mm}$ around the discs with clavulanic acid over the discs with cephalosporin alone confirms ESBL production.

\section{Results}

During the study, 210(53.6\%) males and $182(46.4 \%)$ female samples were obtained from the total of 392 urine samples. Out of 392 samples, total $200(51 \%)$ samples were found to be positive for uropathogen in culture.

Out of $\mathbf{2 0 0}$ positive urine samples for uropathogen, 74(35\%) and $126(69 \%)$ samples were from Male and Female patients, respectively.

Table 2: Age \& Gender wise distribution of UTI patients with POSITIVE cultures

\begin{tabular}{|c|c|c|c|c|c|c|}
\hline Age(Yrs) & Females & $\begin{array}{c}\text { Culture } \\
\text { positive }\end{array}$ & $\mathbf{\%}$ & Males & $\begin{array}{c}\text { Culture } \\
\text { positive }\end{array}$ & $\mathbf{\%}$ \\
\hline $12-20$ & 12 & 9 & 75 & 8 & 0 & 0 \\
\hline $21-30$ & 18 & 15 & 83 & 36 & 8 & 22 \\
\hline $31-40$ & 34 & 30 & 88 & 32 & 8 & 25 \\
\hline $41-50$ & 42 & 34 & 81 & 42 & 18 & 43 \\
\hline $51-60$ & 38 & 30 & 78 & 34 & 14 & 41 \\
\hline$>60$ & 38 & 8 & 22 & 58 & 26 & 45 \\
\hline Total & 182 & 126 & 69 & 210 & 74 & 35 \\
\hline
\end{tabular}


Graph 1: Gender Distribution of culture positive UTI cases

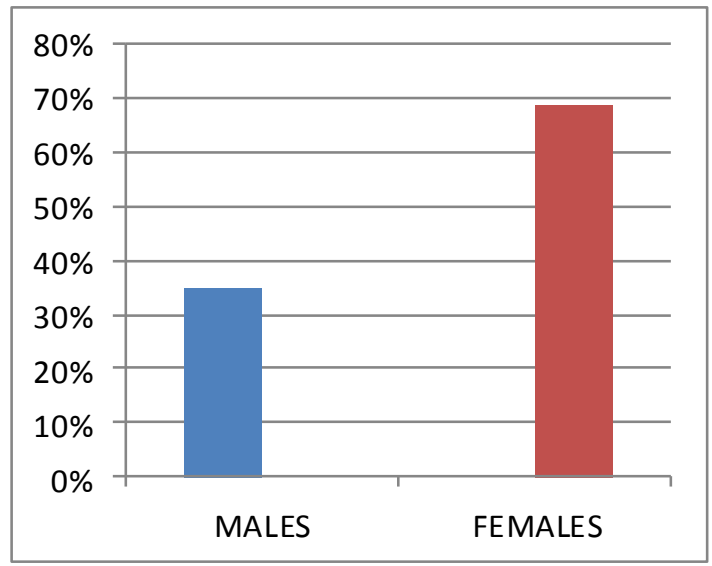

Graph 2: Distribution pattern of uropathogens from the urinary specimens

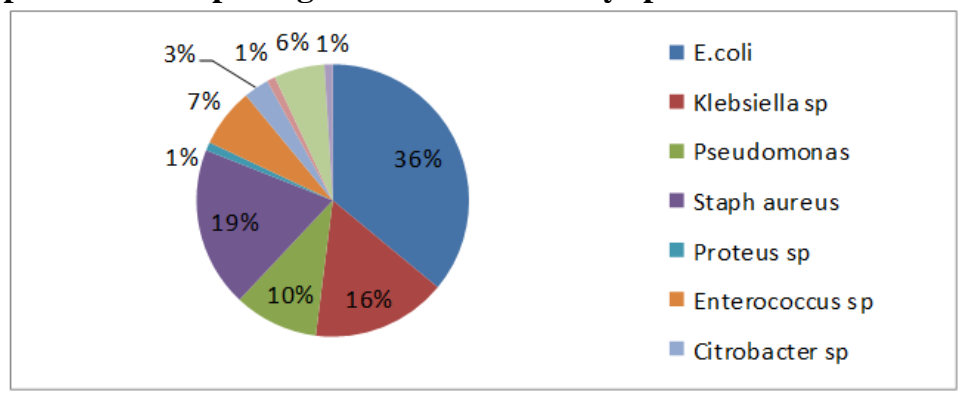

Table 3: Antibiotic susceptibility of Gram Negative isolates

\begin{tabular}{|l|c|c|}
\hline \multicolumn{1}{|c|}{ Antibiotic } & Sensitive (\%) & Resistance (\%) \\
\hline Ciprofloxacin & 43 & 57 \\
\hline Amikacin & 70 & 30 \\
\hline Cotrimoxazole & 40 & 60 \\
\hline Amoxiclav & 32 & 68 \\
\hline $\begin{array}{l}\text { Imipenem }+ \\
\text { cilastin }\end{array}$ & 88 & 12 \\
\hline Colistin & 78 & 22 \\
\hline $\begin{array}{l}\text { Piperacillin }+ \\
\text { Tazobactam }\end{array}$ & 79 & 21 \\
\hline Levoflox & 59 & 41 \\
\hline Nitrofurantoin & 50 & 50 \\
\hline Cefotaxime & 41 & 59 \\
\hline $\begin{array}{l}\text { Cefotaxime }+ \\
\text { clavulinic acid }\end{array}$ & 69 & 31 \\
\hline Aztreonam & 57 & 43 \\
\hline
\end{tabular}

Table 4: Antibiotic susceptibility of Gram positive isolates

\begin{tabular}{|l|c|c|}
\hline \multicolumn{1}{|c|}{ Antibiotic } & Sensitive (\%) & Resistance (\%) \\
\hline Ciprofloxacin & 54 & 46 \\
\hline Azithromycin & 50 & 50 \\
\hline Cotrimoxazole & 70 & 30 \\
\hline Amoxiclav & 46 & 54 \\
\hline Vancomycin & 77 & 23 \\
\hline Colistin & 65 & 35 \\
\hline $\begin{array}{l}\text { Piperacillin }+ \\
\text { Tazobactam }\end{array}$ & 65 & 35 \\
\hline
\end{tabular}




\begin{tabular}{|l|c|c|}
\hline Linezolid & 85 & 15 \\
\hline Nitrofurantoin & 54 & 46 \\
\hline Cefoxitin & 70 & 30 \\
\hline $\begin{array}{l}\text { Ceftazidime }+ \\
\text { clavulinic acid }\end{array}$ & 50 & 50 \\
\hline Ceftazidime & 30 & 70 \\
\hline Ceftriaxone & 70 & 30 \\
\hline
\end{tabular}

Table 5: Prevalence of ESBL Producers

\begin{tabular}{|l|c|c|c|}
\hline \multicolumn{1}{|c|}{ Isolate } & $\begin{array}{c}\text { Total no. } \\
\text { of Isolates }\end{array}$ & $\begin{array}{c}\text { No. of } \\
\text { ESBL'S }\end{array}$ & $\begin{array}{c}\text { \% of } \\
\text { ESBL }\end{array}$ \\
\hline Escherichia coli & 72 & 28 & $38.88 \%$ \\
\hline Klebsiella species & 32 & 8 & $25 \%$ \\
\hline $\begin{array}{l}\text { Pseudomonas } \\
\text { aeruginosa }\end{array}$ & 20 & 6 & $30 \%$ \\
\hline Citrobacter species & 6 & 2 & $33 \%$ \\
\hline Proteus species & 2 & 0 & $0 \%$ \\
\hline $\begin{array}{l}\text { Acinetobacter } \\
\text { species }\end{array}$ & 2 & 0 & $0 \%$ \\
\hline
\end{tabular}

\section{Discussion}

In the present study, it was found that there is a prevalence rate of $51 \%$ culture positivity as compared to $28 \%$ reported by Md. yusuf et al, ${ }^{12} 34 \%$ by singh et $\mathrm{al}^{13}$ and $38 \%$ by ritu. ${ }^{14}$ It goes along parallel with study done by Prakash et $\mathrm{al}^{15}$ which showed $53 \%$ of culture positivity. Differences in positivity rate could be due to media selection, technique of growth and local prevalence rate.

The UTI in Females (69\%) vastly outnumbered to those in Males (35\%). These discrepancies could be related to factors such as the length of urethra, distance of urogenital meatus from anus and antibacterial properties of prostatic fluid. ${ }^{18}$

The young patients are at low risk for occult genitourinary tract abnormalities and are less likely to have co-morbid conditions. Certain patient sub groups, however have complicating conditions that increase the risk for acquiring invasive or systemic infection that include occurrence in men, children and pregnant women. But complications are particularly common in Elderly (61 years or more) particularly in Males (45\%) than Females (22\%), immunocompromised patients and in individuals with neurological disorders, underlying structural abnormalities (e.g. prostate enlargement) and infection due to antimicrobial resistant organisms. ${ }^{20}$

The study demonstrates that among urinary isolates E. coli $(36 \%)$ was predominant uropathogen. Followed by Staphylococcus aureus (19\%) and Klebsiella species $(16 \%)$ in consistent with study done by singh et al. ${ }^{13}$

We also found that among the gram negative urinary isolates ESBL producers were $38.8 \%$ Esch.coli and $25 \%$ Klebsiella as compared to other studies with the total ESBL rate being $32.8 \%$. ESBLs are product of overuse of the $3^{\text {rd }}$ generation Cephalosporins, making it difficult for valid comparison of the prevalence of ESBLs because of variation from hospital to hospital.
These types of discrepancies between the susceptibility data and the disc diffusion results have increased the need for an improved and advanced method of ESBL detection and incorporate it into routine susceptibility procedure.

Amoxiclav (68\%), Co-trimoxazole (60\%) and Cefotaxime (59\%) revealed resistance on higher side to gram negative isolates as correlated with previous studies done. Aminoglycosides, most of them being in injectable form and nephrotoxic are preferred less as the drug of choice and therefore showed less resistance to Esch.coli. Retrospectively, our study should resistance of $57 \%$ to Floroquinolones. ${ }^{24,25}$

This study also revealed high resistance to $3^{\text {rd }}$ generation Cephalosporins, predominantly to Ceftazidime $(70 \%)$ and cefotaxime $(59 \%)$. Over the time there are significant changes in antimicrobial resistance patterns and increase in antimicrobial resistance among uropathogens. Keeping this in view we should have drugs as reserve for last line of treatment which go in combination with inhibitors (like augmentin). ${ }^{27}$

But most probably in near future if this irrational use is not stopped, infection with gram negative organisms will increase the rate of resistance to drugs that are now sensitive resulting in increase morbidity and mortality. ${ }^{27}$

\section{Conclusion}

This study revealed that majority of the urinary isolates obtained from UTI patients are Gram negative. The two principal urinary pathogens are Escherichia coli (gram negative) and Staphylococcus aureus (gram positive). This study indicates that it is essential for prompt recognition of antimicrobial resistant organism. Infection control practitioners and clinicians requires the clinical lab to rapidly identify and characterize 
different types of resistant bacteria especially ESBLs to efficiently minimize the spread of these bacteria and help to select more appropriate antibiotics. Thus strict policy for antibiotic susceptibility testing and prescription should be followed to prevent treatment failure and further emergence of resistant strains.

Funding: No funding sources.

Conflict of interest: None declared.

\section{References}

1. Akram M, Shahid M, Khan AU. Etiology and antibiotic resistance patterns of community-acquired urinary tract infections in J N M C Hospital Aligarh, India. Ann Clin Microbiol Antimicrob 2007;6(1):4

2. Hossain MD, Ahsan S, Kabir MS. Md. Shahidul Kabir. Antibiotic resistance patterns of uropathogens isolated from catheterized and noncatheterized patients in Dhaka, Bangladesh. Tzu Chi Med J 2014;26(3):127-31.

3. Levinson W, Jawetz E. Medical microbiology and immunology: examination and board review. 6th ed. New York: McGraw-Hill; 2000.

4. Zelikovic I, Adelman RD, Nancarrow PA. Urinary tract infections in children. An update. West J Med 1992;157(5):554-61.

5. Sobel JD, Kaye D. Urinary tract infections. In: Mandell GL, Bennett JE, Dolin R, editors. Mandell, Douglas and Bennett's Principles and Practice of Infectious Diseases. 7th ed. Philadelphia: Churchill Livingstone; 2010. pp. 957-85

6. Dimitrov TS, Udo EE, Emara M, Awni F, Passadilla R. Etiology and antibiotic susceptibility patterns of community-acquired urinary tract infections in a Kuwait hospital. Med Princ Pract 2004;13(6):334-39.

7. Bajpai T, Pandey M, Varma M, Bhatambare GS. Prevalence of extended spectrum beta-lactamase producing uropathogens and their antibiotic resistance profile in patients visiting a tertiary care hospital in central India: implications on empiric therapy. Indian $J$ Pathol Microbiol 2014;57(3):407-12.

8. mackie \& mccartney; practical medical microbiology. 14th ed, churchilli livingstone: an implrint of elsevier,Editors: j gerald collee et al., rajkamal electric press: delhi; 2006

9. bauer a.w, kirby w.m.m, sherris j.c et al. Antibiotic susceptibility testing by a standardized single disk Method. Am J Clin Pathol 1966;45:493-96.

10. Clinical and Laboratory Standards Institute (CLSI). Performance standards for antimicrobial susceptibility testing. Twent Informational Suppl 2016;29:1-160.

11. National Committee for Clinical Laboratory Standards. Performance standards for antimicrobial susceptibility testing. 11th ed. Pa: International Supplement. NCCLS Committee for Clinical Laboratory Standards Wayne; 2001.

12. Md. Abdullah Yusu, Afroza Begum and Chowdhury Rafiqul Ahsan. Antibiotic sensitivity pattern of gram negative uropathogenic bacilli at a private hospital in Dhaka city US National Library of Medicine enlisted, J. Al Ameen J. Med. Sci., Volume 8, No.3. Al Ameen J Med Sci 2015;8(3):189-19.
13. Singh Randhir K. Dewasy Bijoylakshmi, Mallick Ram L and Kafle Tara K, Prevalence of antibiotic sensitivity pattern of uropathogens in patients of different agegroups from western region of Nepal. Int J Med Res Health Sci 2016;5(9):1-7.

14. Ritu Agarwal, Maneesh Goyal, Dakshina Bisht, Rinku Garg, Bacterial isolates and their antibiotic sensitivity profile recovered from urine samples in NCR, Ghaziabad (Uttar Pradesh). J Evolution Med Dent Sci 2014;3(28).

15. Prakash D, Saxena RS. Distribution and antimicrobial susceptibility pattern of bacterial pathogens causing urinary tract infection in urban community of Meerut city, India, ISRN Microbiol Volume 2013.

16. Dash M, Padhi S, Mohanty I, Panda P, Parida B. Antimicrobial resistance in pathogens causing urinary tract infections in a rural community of Odisha, India. $J$ Family Community Med 2013;20(1):20-6.

17. Patel R, Chavada F. Study Of Bacteriological And Antimicrobial Susceptibility Pattern Of Uropathogens. Natl J Integr Res Med 2016;7(1):47-9.

18. Omoregie R, Erebor JO, Ahonkhai I, Isobor JO, Ogefere HO. Observed changes in the prevalence of uropathogens in Benin City, Nigeria. NZJ Med Lab Sci 2008;62:29-31.

19. Akram M, Shahid M, Khan AU. Etiology and antibiotic resistance patterns of community acquired urinary tract infections in JNMC Hospital, Aligarh, India. Ann Clin Microbiol Antimicrob 2007;6:6.

20. Das RN, Chandrashekhar TS, Joshi HS, Gurung M, Shrestha N, Shivananda PG. Frequency and susceptibility profile of pathogens causing urinary tract infections at a tertiary care hospital in western Nepal. Singapore Med J 2006;47(4):281-85.

21. Pitout JD, Nordmann P, Laupland KB, Poirel L. Emergence of Enterobacteriaceae producing extendedspectrum $\beta$-lactamases (ESBLs) in the community. $J$ Antimicrob Chemother 2005;56(1):52-9.

22. Aggarwal R, Chaudhary U, Sikka R. Detection of extended spectrum $\beta$-lactamase production among uropathogens. J Lab Physicians 2009;1(1):7-10.

23. Sood S, Gupta R. Antibiotic resistance pattern of community acquired uropathogens at a tertiary care hospital in jaipur, rajasthan. Indian J Community Med 2012;37(1):39-44.

24. Ebie MY, Kandaki-Olukemi YT, Ayanbadejo J, Tanyigna KB. UTI infections in a Nigerian Military Hospital. Nigerian J Microbiol 2001;15(1):31-7.

25. Ehinmidu JO, Bolaji RO, Adegboye EE. Isolation and antibiotic susceptibility profile of Neisseria gonorrhoeae isolated from urine samples in Zaria, northern Nigeria. $J$ Phytomed Therapeutics 2003;8-11:20-4.

26. Burbige KA, Retic AB, Colony A, Bauer SB, Lebowitz R. UTI in boys. J Urol 1984;132:541-2.

27. Manjunath GN, Prakash R, Annam V, Shetty K. The changing trends in the spectrum of the antimicrobial drug resistance pattern of uropathogens which were isolated from hospitals and community patients with urinary tract infections in Tumkur and Bangalore. Int J Biol Med Res 2011;2(2):504-50.

How to cite this article: Khaleel M., Khan R., Samreen S., Arshi S. Detection of Extended spectrum beta lactamases in gram negative isolates causing urinary tract infection in Tertiary care centre. Int J Med Microbiol Trop Dis 2018;4(4):234-39. 